\title{
Traumatic Bilateral Mainstem Bronchial Avulsion in a Red Fox (Vulpes vulpes)
}

\author{
Seth M. Ganz, DVM ${ }^{1}$, Susan L. Bartlett, DVM ${ }^{2}$, Mauricio Solano, MV, ACVR ${ }^{3}$, \\ Florina S. TSEnG, DVM ${ }^{4}$, Mark A. Pokras, DVM ${ }^{4}$ \\ ${ }^{1}$ Veterinary Specialty Hospital, San Diego, California \\ ${ }^{2}$ Cornell University, ItHACA, New York \\ ${ }^{3}$ Tufts Cummings School of Veterinary Medicine, North Grafton, Massachusetts \\ ${ }^{4}$ Tufts Wildlife Clinic, North Grafton, Massachusetts
}

\begin{abstract}
An adult male red fox (Vulpes vulpes) was hit by an automobile and presented in respiratory distress. Radiographs and a physical examination indicated that both bronchi had been avulsed from the lungs and euthanasia was elected.

This is the first report of a bilateral bronchial avulsion, as well as the first report of large airway avulsion in a canid, as a result of blunt thoracic trauma. Tracheal avulsion is a sporadically reported sequel to blunt trauma in domestic cats. Preliminary diagnosis can be made from history, clinical signs, and radiographs. Bronchoscopy yields a definitive diagnosis. Treatment is most often surgical resection of traumatized ends of airway and anastamosis. Depending on the location and extent of injury, other surgical techniques may be considered.
\end{abstract}

An index of suspicion for this injury must be maintained in any patient suffering blunt trauma, as patients with this severe injury may present with mild signs and initially respond to conservative therapy. Thoracic radiographs should be obtained promptly in all cases of suspected blunt thoracic trauma.

Keywords: red fox, Vulpes, trauma, bronchial avulsion, respiratory distress

Seth Ganz graduated from Tufts Cummings School of Veterinary Medicine in 2005. He is currently with the Veterinary Specialty Hospital in San Diego, CA.

Susan Bartlett received her DVM from Cornell University in 2003 and completed an internship with the Tufts Wildlife Clinic in 2005. She is currently a resident in zoological medicine at Cornell University.

Mauricio Solano received his veterinary degree (MV) at the Universidad de LaSalle in Bogotá, Colombia in 1986. Dr. Solano completed a residency in radiology at Tufts University, where he currently works as an assistant professor of radiology.

Florina Tseng graduated from Cornell's College of Veterinary Medicine in 1981 and did an internship at Wildlife Center of Virginia. She is currently an Assistant Professor at the Wildlife Clinic at Tufts Cummings School of Veterinary Medicine and Assistant Director of Tufts Wildlife Clinic. She is a former member of the NWRA Board of Directors.

Mark Pokras graduated from Tufts School of Veterinary Medicine in 1984 and also did a residency in wildlife and zoologic medicine there. He is Director of the Tufts Wildlife Clinic and serves as Director of the Center for Conservation Medicine.

\section{INTRODUCTION}

An adult male red fox (Vulpes vulpes) weighing $4.8 \mathrm{~kg}$ presented to the Tufts University School of Veterinary Medicine (TCSVM) wildlife clinic approximately one hour after being struck by a motor vehicle.

\section{DiAgNOSTICS}

On presentation, the fox was minimally responsive and in respiratory distress. Abnormal physical examination findings included bilateral epistaxis, swelling over the left temporal region, and an abrasion under the rostral mandible. Mucous membranes were slightly pale with a capillary refill time of approximately two seconds, and respirations were weak with an abdominal component and pronounced end-expiratory stertor. Rectal temperature was $96.8^{\circ} \mathrm{F}\left(36^{\circ} \mathrm{C}\right)$. Right lateral and dorsoventral whole body and head and neck radiographs were obtained while the fox received 100 percent oxygen by mask. Initial treatment included administration of lactated Ringer's solution (114 ml $\mathrm{SC}$ ), carprofen (2.8 mg/kg SC), enrofloxacin ( $2.5 \mathrm{mg}$ / $\mathrm{kg}$ IM), and placement in an oxygen chamber.

Radiographs. Radiographic findings (Figures 1 and 2) include a discontinuity of the tracheal lumen approximately $8 \mathrm{~mm}$ distal to the carina. In addition, there is dorsal displacement of the thoracic portion of the trachea and an approximately $28 \mathrm{~mm}$ separation of the proximal airway segment from the distal segment. A soft tissue opacity measuring 13 $\mathrm{x} 25 \mathrm{~mm}$ is present in the region of the pulmonary parenchyma between the displaced airway segments. Free gas accumulation can be seen dorsal and ventral to the trachea, allowing increased visualization of the tracheal walls and other mediastinal structures. Mediastinal widening is noted in the dorsoventral view and increased soft tissue opacity ventral to the 


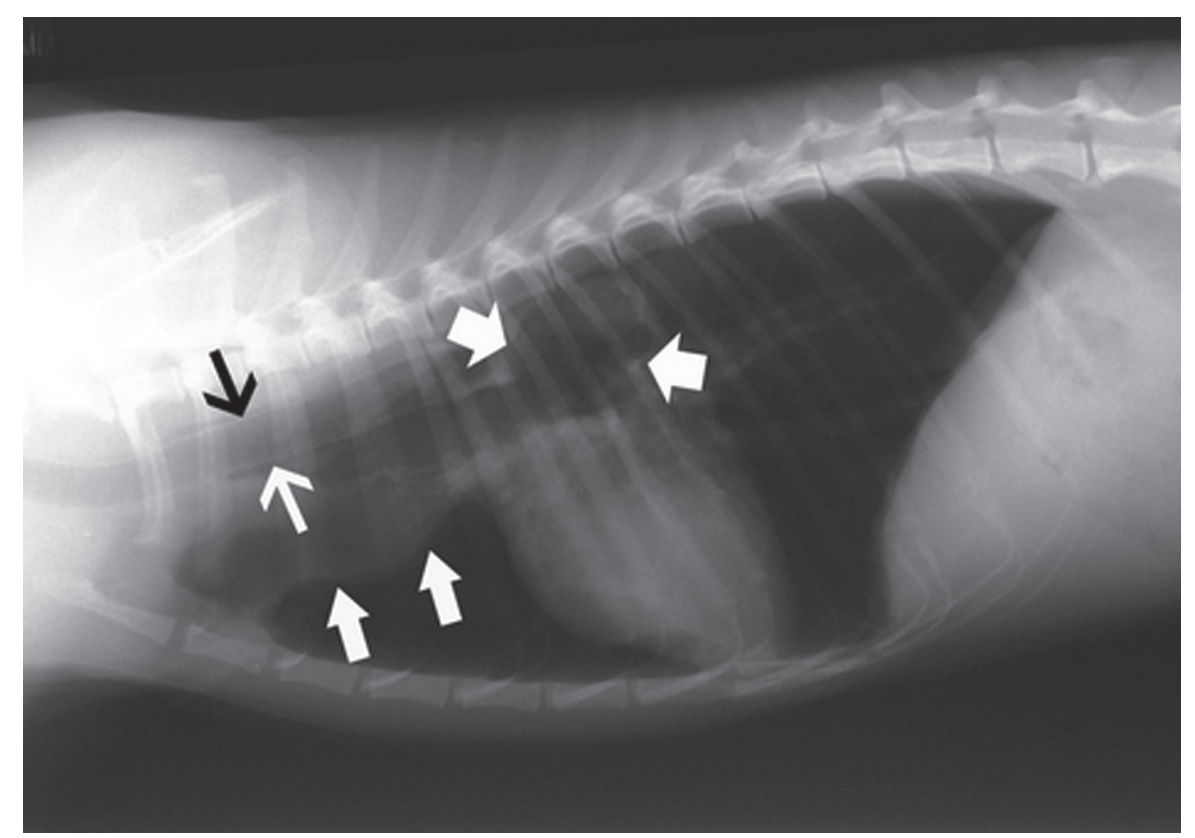

Figures 1 E 2. Right lateral (Figure 1) and dorsoventral (Figure 2) thoracic radiographs. Note abnormal visualization of the tracheal wall (thin arrows), soft tissue/fluid density in the cranial mediastinum (medium arrows), and discontinuity of the distal large airway (wide arrows). Also note decreased sternal contact of the cardiac silhouette and flattening of the diaphragm (lateral view) and left scapular and right 1st rib fractures (dorsoventral view).

\section{LITERATURE REVIEW} AND DISCUSSION

Intrathoracic tracheal avulsion in domestic cats has been reported sporadically in the veterinary literature (Barber and Rawlings 1981; Brouwer et al 1984; Feeney et al 1979; Griffiths et al 1998; Kennedy 1976; Ryan and Smith 1972; White and Burton 2000; White and Milner 1995; Zerenner et al 2003), with a total of 22 cases documented. This is the first report of a bilateral bronchial avulsion, as well as the first report of large airway avulsion in a canid, as a result of blunt thoracic trauma. There has been one report of unilateral principal bronchus avulsion in a cat as a result of suspected blunt trauma (White and Oakley 2001). With the exception of one documented case secondary to trachea is detected on the lateral view. There is decreased sternal contact of the cardiac silhouette and flattening of the diaphragm on the lateral view. Comminuted, non-articular left scapular and right first rib fractures are also present.

Radiographic findings were compatible with a large airway avulsion distal to the carina, pneumomediastinum, fluid in the mediastinum, and pneumothorax. The pulmonary opacity noted is suggestive of pulmonary contusion, traumatized peri-bronchial tissue, or a combination thereof. Lateral and dorsoventral radiographic views of the abdomen, head, and neck showed no abnormalities.

Post Mortem Findings. Based upon the presumptive diagnosis of complete large airway avulsion and concurrent injuries, the fox was euthanized. Necropsy revealed complete avulsion of both mainstem bronchi approximately $8 \mathrm{~mm}$ distal to the tracheal bifurcation (Figure 3). There was no adventitial membrane bridging the avulsed ends. Blood was present in the mediastinum.

An approximately $2 \mathrm{~cm}$ laceration was present in the descending aorta at the level of the airway avulsion. The scapular and rib fractures were confirmed.
Figure 2.

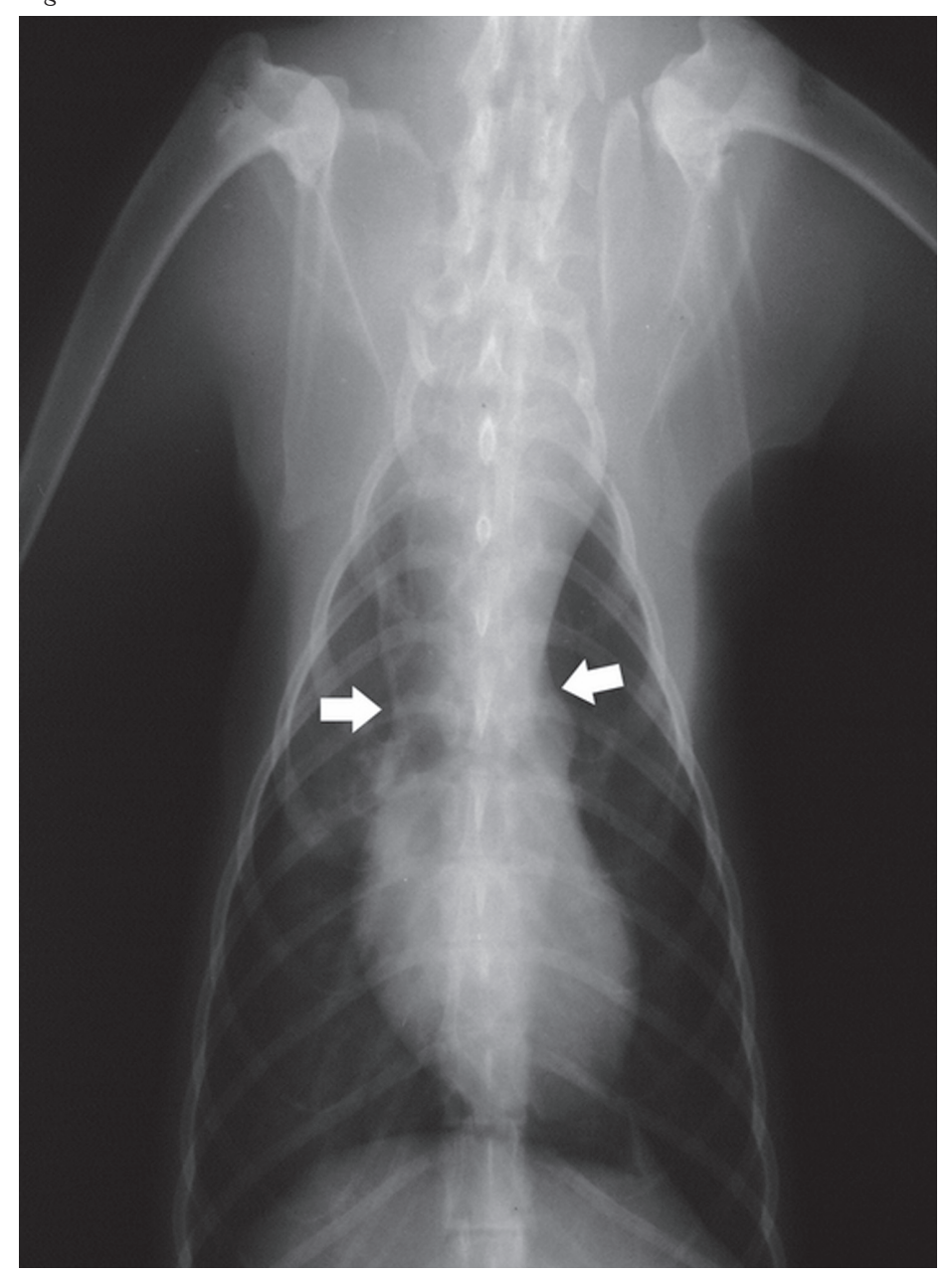




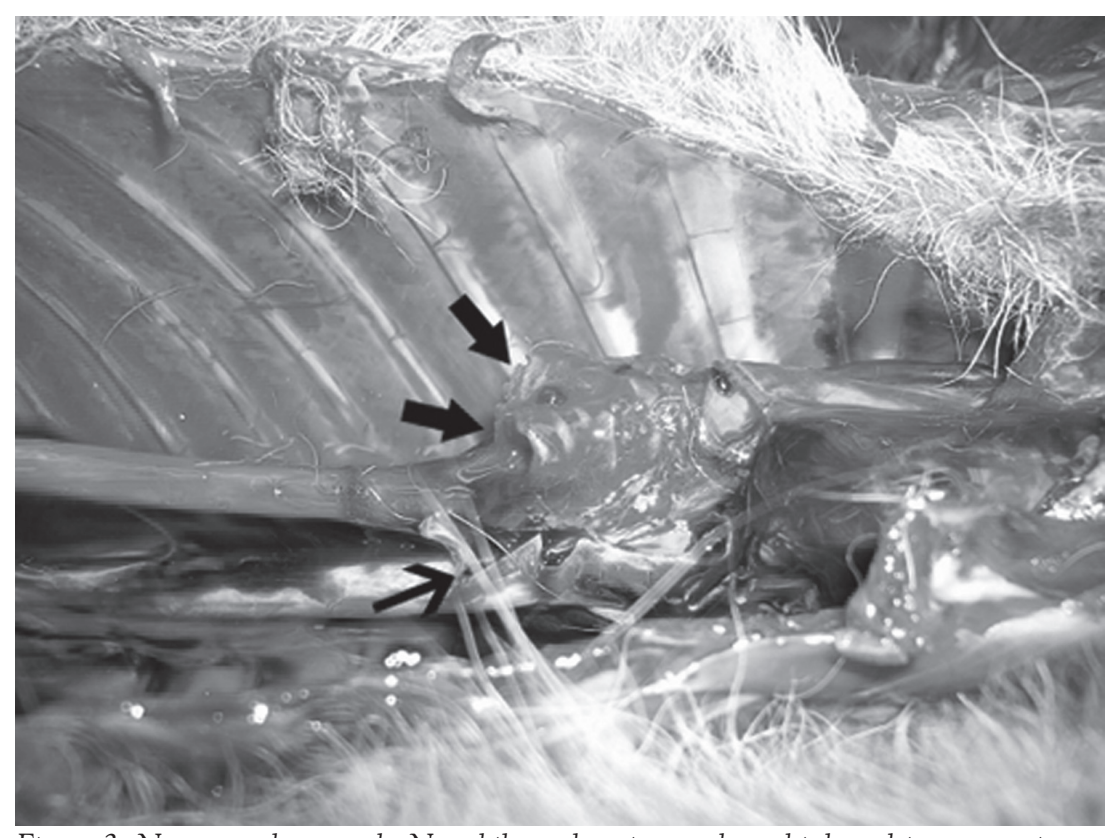

Figure 3. Necropsy photograph. Note bilateral mainstem bronchial avulsion approximately $8 \mathrm{~mm}$ distal to the tracheal bifurcation (medium arrows) and aortal laceration (thin arrow).

direct blunt injury to the cervical trachea (Kellagher and White 1987), reported cases of large airway avulsion in the dog have been as the result of penetrating injury or disease. Previous reports of intrathoracic tracheal avulsion in cats describe two basic clinical scenarios. The more common clinical course is that of a cat suffering known trauma (most often a motor vehicle accident) and being treated conservatively with initial success. These patients inevitably re-present days to weeks later for respiratory compromise. The other, less common clinical course is that of acute respiratory distress following a traumatic episode. The cats in the former group maintain clinically normal respiration/ventilation due to bridging of the avulsed ends by tracheal adventitia, the resulting structure being alternatively referred to as a pseudotrachea (White and Burton 2000), pseudodiverticulum (Griffiths et al 1998), or more generally a pseudoairway (White and Burton 2000; White and Milner 1995; Griffiths et al 1998).

In human studies, tracheal adventitia has been found to consist of a loose outer layer and dense inner layer of connective tissue that joins the annular ligament to allow the trachea some tolerance to deformation (Ohkimoto et al 1997). The subsequent respiratory decompensation that occurs in cats appears to result from progressive stenosis of the avulsed ends of the trachea, severely limiting airflow to the lungs. Cats in the latter group do not possess a pseudotrachea when examined in surgery or at necropsy. Pneumomediastinum is evident on thoracic radiographs in these cases. The mediastinum may itself temporarily act as a pseudoairway, but these cats are often compromised more severely than those with a pseudotrachea. It is thought possible that those cats that initially responded to medical therapy could in fact have had a pneumomediastinum at presentation since radiographs were not initially obtained in these cases (Griffiths et al 1998). It has also been theorized that the pseudotrachea itself forms as a result of the turbulent airflow that develops secondary to stenosis of the avulsed tracheal ends (White and Oakley 2001). If this is true, it would imply that in patients that present in acute distress, lack of pseudotrachea is not the cause of the severity of signs, but simply an earlier stage of the condition that is recognized when imaging, surgery, or necropsy are performed early on in the course. Therefore, the pseudotrachea should not necessarily be considered a life-saving structure, as the mediastinum may serve as an effective conduit of air for days to weeks until the avulsed ends stenose and produce a pseudotrachea.

A presumptive diagnosis of large airway avulsion can often be made based on radiographic evidence of tracheal discontinuity either with a bullous structure bridging the discontinuous ends or with the appearance of pneumomediastinum (Barber and Rawlings 1981; Brouwer et al 1984; Feeney et al 1979; Griffiths et al 1998; Ryan and Smith 1972; White and Burton 2000; White and Milner 1995). If the pseudoairway only forms as a result of airflow changes secondary to stenosis, one may not expect to find this feature on initial radiographic study. Definitive diagnosis can be obtained via bronchoscopy. Depending on the time from injury, one would recognize bronchoscopically a discontinuity/separation of tracheal rings, pseudotrachea, or intraluminal presence of a sagging dorsal tracheal membrane (White and Burton 2000; White and Milner 1995).

Regardless of the clinical course, surgical management is ultimately warranted and most often consists of resection of the compromised and/or necrotic avulsed ends of the trachea (usually 6 to 8 rings) and anastamosis (Fingland et al 1995; Hedlund 1984; Berg et al 1991). The surgery is most commonly performed via a right lateral thoracotomy through the 3rd or 4th intercostal space (White and Burton 2000; Berg et al 1991). Studies in dogs indicate that resection using 
a tracheal ring splitting technique (Hedlund 1984) and anastamosis using a simple continuous suture pattern (Fingland et al 1995) is preferred to maintain strength and minimize luminal stenosis. In cases in which resection and anastamosis are not feasible due to location (i.e., carinal avulsion) or extent of resection required, balloon dilation may be an appropriate therapy. This procedure is also done via a right lateral thoracotomy and has been combined with postoperative corticosteroid therapy achieving successful long-term results (Berg et al 1991). A reliable, relatively simple and inexpensive surgical treatment for unilateral avulsions distal to the carina is lung lobectomy. Careful anesthetic management is vital for any of these procedures. Although different protocols have been attempted to maintain ventilation during the resection and anastamosis, a recently reviewed method (White and Burton 2000) involves intubation with an uncuffed endotracheal tube, placement of a cuffed tube in the distal segment through the thoracotomy immediately following transection of the stenosed end of the distal segment, and finally replacing the original uncuffed tube through both segments following transection of the stenosed end of the proximal segment. This appears to be a simple and reliable method for maintaining ventilation with little interruption.

The mechanism of injury in cases of traumatic tracheal avulsion has not been definitively established. The avulsion is thought to occur by several mechanisms in different combinations (Brouwer et al 1984; Feeney et al 1979; Griffiths et al 1998; White and Burton, 2000; White and Milner 1995; Berkwitt and Berzon 1985). The distal trachea and lower airways have a different mobility and specific gravity than does the proximal thoracic trachea. When the body experiences blunt force, these structures are accelerated differentially, producing shearing forces. These shearing forces can be exacerbated by hyperextension of the neck, which fixes the proximal thoracic trachea while exerting a distractive force on the distal airway. These forces appear to exceed the tolerance of the annular ligament and trachealis muscle most commonly at a level 1 to $4 \mathrm{~cm}$ cranial to the tracheal bifurcation, where the majority of reported injuries have occurred (Barber and Rawlings 1981; Brouwer et al 1984; Feeney et al 1979; Griffiths et al 1998; Kennedy 1976; Ryan and Smith 1972; White and Burton 2000; White and Milner 1995; Zerenner et al 2003). If hyperextension is severe enough, it is thought that the resulting distractive force on the trachea alone can produce an avulsion that could be located more distally (Griffiths et al 1998). Finally, dramatic increases in airway intraluminal pressure caused by impact forces on the abdominal and/or thoracic cavities with a closed glottis have been proposed as a contributing factor.

Although the road traffic accident was witnessed in this case, the orientation of the fox relative to the vehicle at impact was not determined. The scapular and rib fractures and pneumothorax coupled with the mandibular, temporal and bronchial injuries suggest a combination of blunt trauma perhaps to the fox's left side (left scapular fracture, left temporal swelling) and severe neck hyperextension (rostral mandibular abrasion and described intrathoracic injuries). This type of impact is likely to have produced a combination of all of the forces discussed above. It is theorized that the dominant force on the airway causing the avulsion at such a distal location in this case was distractive and a result of neck hyperextension.

The fox in this report had pneumomediastinum diagnosed on thoracic radiographs, and there was no true pseudoairway found on necropsy. Cats in previous reports of tracheal avulsion that had a history of acute, severe respiratory compromise had a pneumomediastinum and no pseudotrachea (Griffiths et al 1998). The principle bronchi do possess an adventitia and a pseudoairway has been observed following unilateral bronchial avulsion in a previous report (White and Oakley 2001). The lack of such a structure on necropsy in this case, therefore, is consistent with its history of acute, severe respiratory compromise. It is presumed that the fox in this report was able to maintain temporarily sufficient ventilation after the avulsion via the mediastinum. The appearance of fluid in the mediastinum on radiographs and blood in the mediastinum on necropsy are consistent with vessel trauma. The defect observed in the aorta on necropsy appeared to have been too large to account for the moderate accumulation of blood in the mediastinum and should not have been compatible with life. It is unknown whether a smaller aortic laceration/avulsion was present at the time of euthanasia and was iatrogenically enlarged subsequently or whether the entire defect was created at necropsy. The defect was, however, present at the level of the avulsion.

\section{CONCLUSION}

This case represents the first report of bilateral traumatic bronchial avulsion, as well as the first report of large airway avulsion as a result of blunt thoracic trauma in a canid. In previous reported cases of traumatic intrathoracic tracheal avulsion in cats, external injuries were often surprisingly minimal, and the patients' overall level of distress most often mild to moderate. In this report, although the patient was mentally 
depressed, and although the respiratory pattern was abnormal, he was relatively stable when placed in oxygen. This emphasizes the need for a certain index of suspicion for this injury, especially if the patient is not responding to medical therapy as expected, and highlights the importance of obtaining thoracic radiographs in any patient known to have experienced significant blunt trauma. Radiographic evidence of this injury could be subtle at first, and one should consider repeat radiographs or bronchoscopy in patients without obvious radiographic evidence of airway avulsion in which response to initial therapy is disappointing. Although this particular injury is thought to be rare, airway avulsion should be considered a possible diagnosis in any patient known to have suffered blunt trauma and having respiratory distress at any point in time after presentation.

\section{LITERATURE CITED}

Barber, D. L., and C. A. Rawlings. 1981. Radiographic diagnosis. Veterinary Radiology. 22:258-261.

Berg, J., C. R. Leveille, and M. W. O'Callaghan. 1991.

Treatment of post-traumatic carinal stenosis

by balloon dilation during thoracotomy in a cat. Journal of the American Veterinary Medical Association. 198:1025-1027.

Berkwitt, L., and J. L. Berzon. 1985. Thoracic trauma-newer concepts. Veterinary Clinics of North America, Small Animal Practice. 15:1031-1039.

Brouwer, G. J., H. M. Burbidge, and D. E. Jones. 1984. Tracheal rupture in a cat. Journal of Small Animal Practice. 25:71-76.

Feeney, D. A., G. Malnati, and M. B. Mahaffey. 1979. Ruptured trachea in a cat (What is your diagnosis?). Journal of the American Veterinary Medical Association. 175:303-304.

Fingland, R. B., et al. 1995. A comparison of simple continuous versus simple interrupted suture patterns for tracheal anastamosis after large-segment tracheal resection in dogs. Veterinary Surgery. 24:320-330.

Griffiths, L. G., M. Sullivan, and P. Lerche. 1998. Intrathoracic tracheal avulsion and pseudodiverticulum following pneumomediastinum in a cat. Veterinary Record. 142:693-696.

Hedlund, C. S. 1984. Tracheal anastamosis in the dog; comparison of two end-to-end techniques. Veterinary Surgery. 13:135-142.

Kellagher, R. B., and R. S. White. 1987. Tracheal rupture in a dog. Journal of Small Animal Practice. 28:29-38.
Kennedy, R. K. 1976. Traumatic tracheal separation with diverticuli in a cat. Veterinary Medicine, Small Animal Clinician. 71:1384-1385.

Ohkimoto, K.., M. Mouri, M. Amatsu, and M. Teraoka. 1997. Histological study of the tracheal adventitia, perichondrium and annular ligament. Nippon Jibiinkoka Gakkai Kaiho. 100(11):1394-400. (Abstract-article in Japanese).

Ryan, C. O., and R. A. Smith. 1972. Separation of tracheal rings in a cat (What is your diagnosis?). Journal of the American Veterinary Medical Association. 161:1151-1152.

White, R. N., and M. R. Oakley. 2001. Left principle bronchus rupture in a cat. Journal of Small Animal Practice. 42:495-498.

White, R. N., and C. A. Burton. 2000. Surgical management of intrathoracic tracheal avulsion in cats: long-term results in 9 consecutive cases. Veterinary Surgery. 29(5):430-435.

White, R. N., and H. R. Milner. 1995. Intrathoracic tracheal avulsion in three cats. Journal of Small Animal Practice. 36:343-347.

Zerenner, D. M., K. A. Agnello, and C. A. Carberry. 2003. Tracheal avulsion in a cat (What is your diagnosis?). Journal of the American Veterinary Medical Association. 222:1683-1684. (․ㅏㅇ

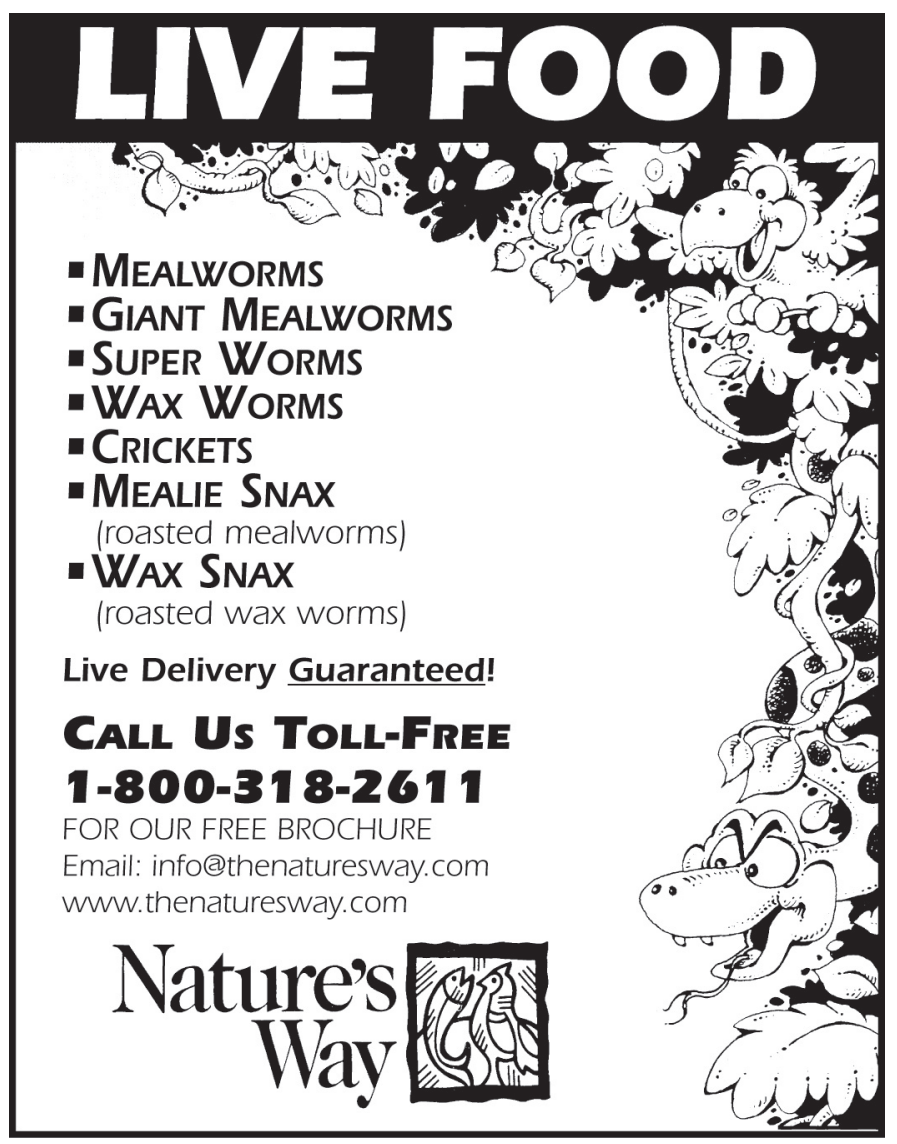

